\title{
TEXTSORTENKLASSIFIKATION AUF DER BASIS KOMMUNIKATIVER GRUNDBEDINGUNGEN
}

\section{Zielsetzung}

Im folgenden wird versucht, eine Basisklassifikation von Textsorten zu entwerfen, die umfassend und nichtwillkürlich ist. Mit "umfassend" ist gemeint, daß nicht nur ein bestimmter Ausschnitt aus dem Spektrum möglicher Sprachverwendungen untersucht wird, sondern daß alle Vorkommen dominant sprachlicher Kommunikation, mit denen ein Sprecher normalerweise konfrontiert ist, einbezogen werden. ${ }^{1}$ Mit "nichtwillkürlich" ist zum einen gemeint, daß die Wahl der Klassifikationskriterien nicht beliebig, sondern aus relevanten, textexternen Faktoren ableitbar ist. ${ }^{2}$ Zum anderen betrifft das Problem der Willkürlichkeit die Frage, ob ein Modell die schlüssige Zurückführung von Texteigenschaften auf entsprechende Klassifikationsmerkmale ermöglicht. ${ }^{3}$

Die hier herangezogenen Klassifikationsmerkmale sind aus denjenigen situativen Faktoren abgeleitet, die die kommunikativen Grundbedingungen jeder Sprachverwendung bilden, so daß man von ihrem universellen Charakter ausgehen kann. Diese kommunikativen Grundbedingungen sind für jedes Textvorkommen konstitutiv, und ihre unterschiedlichen Ausprägungen hinterlassen eindeutige Reflexe im Text. Die auf diese Weise entstehende Basisklassifikation teilt die Gesamtheit der Texte in wenige Großgruppen, die ich Grundtextsorten nenne. Der Terminus "Grundtextsorte" wird nicht in Opposition zu "Textsorte" gebraucht, er besagt nur, daß außer den Merkmalen der situativen Ebene keine weiteren Merkmale spezifiziert sind. Im folgenden werden die kommunikativen Grundbedingungen und die daraus ableitbaren situativen Merkmale genauer bestimmt und es wird gezeigt, welche Grundtextsorten sich aus den situativen Merkmalen ergeben und welche Texteigenschaften direkt von den situativen

1 Für das Bemühen um eine Gesamtklassifikation plädiert auch Weigand 1986.

2 Kritik an der Beliebigkeit und Willkürlichkeit vieler Klassifikationsversuche übt z. B. Ehlich 1986, der auch einen kurzen Überblick über die Textsortenforschung bietet. Weitere Forschungsüberblicke finden sich in Adamzik 1991, Diewald 1991, Franke 1991 und - sehr ausführlich - in Rolf (1993:81-124).

3 Eine diesbezügliche Kritik bringt z. B. Lux (1981:119) gegenüber dem Freiburger Modell vor; er meint, daß dort "eine Determination der Textsorte durch den Situationstyp [...] lediglich behauptet" werde. 
Merkmalen beeinflußt sind. Vorher sind jedoch einige grundsätzliche Anmerkungen zur Textsortenklassifikation am Platze.

\section{Probleme der Textsortenklassifikation}

Eine Gesamtklassifikation aller Textvorkommen nach Maßgabe der Ausprägung verschiedener kommunikativer Grundbedingungen ist notwendig deduktiv. Das Ergebnis ist eine Klassifikation nach Grundtextsorten, d. h. die Einteilung aller Texte in idealisierte Großgruppen, die von vielen Einzelmerkmalen abstrahieren. Damit befundet sich dieser Ansatz auf den ersten Blick im Widerspruch zu den meisten aktuellen Forschungsbemühungen. Diese betonen im allgemeinen den nicht vorhersagbaren, historisch, sozial und konventionell bedingten Charakter von Textsorten - also die Unberechenbarkeit ihrer Merkmalskombinationen - und konzentrieren sich daher auf die empirische Forschung (z. B. Adamzik 1991:104ff., Fröhlich 1990, Harnisch 1990).

Ich denke, daß diese im Bereich der Textsortenforschung besonders ausgeprägte Spaltung in deduktiv-theoretische und induktiv-empirische Klassifikationsansätze (dazu auch Ehlich 1986, Adamzik 1991) überwunden werden kann, da sie sich auf den - offenbar unbemerkt gebliebenen, aber entscheidenden - Unterschied zwischen System und Norm zurückführen läßt. Textsorten als historisch-konventionelle Textmuster sind Ausprägungen der sprachlichen Norm im Sinne Coserius, also dessen, "was tatsächlich gesagt wird und worden ist, beziehungsweise die Kenntnis einer traditionellen Realisierung". Diese Norm ist die konkrete Realisierung des Systems, das Coseriu bestimmt als das, "was man in einer Sprache sagen kann, ohne ihre Funktionalität anzutasten" (1974:116f.).

Die Einteilung aller Textvorkommen in Grundtextsorten ist zu verstehen als eine Annäherung an die Beschreibung des zugrundeliegenden Systems, das m. E. aus dem grundsätzlichen Funktionieren sprachlicher Kommunikation deduziert werden kann. Die Einteilung in Grundtextsorten bildet die Voraussetzung für alle sich individuell und unvorhersehbar entfaltenden Textsortennormen. Die Erstellung einer solchen Basisklassifikation ist $\mathrm{m}$. E. als Grundlage oder - wenn man so will - als Vorarbeit für jede differenziertere Textsortenklassifikation nötig. Damit ist zugleich gesagt, daß mit der Rekonstruktion der grundlegenden Oppositionen die Klassifikationsarbeit nicht beendet ist, daß man also in der Tat nur die Grundtypen sprachlicher Kommunikationsmöglichkeiten, die Grundtextsorten, erhält. Diese Arbeit muß jedoch vor jeder feineren Differenzierung getan werden, wenn eine einheitliche, nichtarbiträre Textsortenklassifikation angestrebt wird.

Viele Klassifikationen gehen nicht von kommunikativen Grundbedingungen, sondern von Alltagsbezeichnungen für Textsorten oder von Textfunktionen als Klassifikationsbasis aus. Für das hier verfolgte Ziel einer integrativen Gesamtklassifikation ist dieser Weg nicht gangbar. Alltagsbezeichnungen für Textsorten, also umgangssprach- 
liche Textsortennamen, können m. E. nicht als Ausgangspunkt der Suche nach Klassifikationskriterien verwendet werden, ${ }^{4} \mathrm{da}$ oft außerlinguistische Kriterien maßgebend für die alltagssprachliche Benennung sind. Gerade die zugrundeliegenden Kategorien und Oppositionen des Sprachsystems, in diesem Falle also die Merkmalsausprägungen der kommunikativen Grundbedingungen, werden meist nicht benannt, da sie als selbstverständlich vorausgesetzt werden. Oder sie sind - wie ein großer Teil unseres sprachlichen Wissens - den Sprachbenutzern nicht bewußt und daher auch nicht formulierbar. ${ }^{5}$ Für eine Textsortenklassifikation, verstanden als Rekonstruktion der Kompetenz, bedeutet dies, daß das Alltagswissen der Sprecher über Textsorten (wie es in Textsortennamen, Metadiskussion und didaktischer Vermittlung zum Ausdruck kommt) keinen eindeutigen Aufschluß über die fundierenden Merkmale liefert.

Was nun funktionale Kriterien betrifft, so sind auch sie zur Erstellung einer Basisklassifikation nicht geeignet. Unter der Funktion eines Textes wird im allgemeinen das objektivierte Gegenstück der Intention des Produzenten verstanden (s. Rolf 1993:25), d. h. die Klassifikation ruht auf einer Einteilung der Texte nach Sprechakttypen (z. B. Franke 1987, Rolf 1993). Nun sind aber, anders als im Anschluß an Searle oft angenommen wird, Sprechaktbezeichnungen und -klassifikationen - und damit auch alle davon abgeleiteten Textsortenbezeichnungen - nicht universell, sondern kulturspezifisch. Wierzbicka 1991 stellt das deutlich heraus (150ff., 252) und betont, daß gerade auch Sprechakttypen wie "befehlen" oder "versprechen", die oft als universell veranschlagt werden, kulturspezifisch sind (151f.). Eine Textsortenklassifikation, die auf Sprechakttypen aufbaut, analysiert also die kultur- und einzelsprachspezifischen Textsortennormen, nicht jedoch die universellen Merkmale der Grundtextsorten.

Funktional orientierte Klassifikationsansätze haben ein weiteres, schwerwiegendes Problem: Wenn der Funktion klassifikatorische Kraft zukommen soll, dann muß jedem Text eine dominierende Funktion bzw. Intention zugeordnet werden. Während bei monologischen Texten meist eine Hauptfunktion zu finden bzw. zu interpolieren ist, sind dialogische Texte auf diese Weise nicht zu beschreiben, da sie ja per definitionem aus dem Wechselspiel von zwei Partnern und folglich zwei Intentionen aufgebaut sind. Funktional orientierte Klassifikationen - so z. B. Rolf 1993 - beschränken sich daher in der Regel auf die Untersuchung monologischer Texte.

4 Anders Adamzik (1991:107). Dimter 1981 unternimmt einen solchen Klassifikationsversuch auf der Basis umgangssprachlicher Textsortennamen (ausführlich besprochen in Diewald 1991). Ehlich (1986:53f.) rät insgesamt zur Vorsicht bei der Einbeziehung von Alltagswissen und weist darauf hin, daß eine Klassifikation auf der Grundlage von Alltagsbezeichnungen einer Vermischung von Objekt-und Metasprache gleichkommt (1986:60).

5 Aus diesem Grunde kann auch der Versuch von Gülich 1986, Textsortenmerkmale aus metasprachlichen Äußerungen von Sprechern über Textsorten zu gewinnen, nicht zur Erstellung einer Klassifikationsgrundlage dienen. Entsteht doch auch hier - neben der Schwierigkeit der Materialgewinnung - das Problem, daß Textsortenwissen nur in Konfliktfällen, nicht jedoch bezogen auf die grundlegenden Prototypen, expliziert wird. 
Die Funktion ist also - zumindest beim jetzigen Forschungsstand - weder universell bestimmbar, noch kann sie auf alle Verwendungsarten von Sprache als primäres Differenzierungskriterium angewendet werden. Sie ist daher als Basis für eine integrative Gesamtklassifikation nicht geeignet. Für diesen Zweck kommen nur situative Merkmale in Frage, die im folgenden als kommunikative Grundbedingungen genauer zu bestimmen sind.

\section{Die kommunikativen Grundbedingungen}

In Anlehnung an den Freiburger Ansatz, wie er z. B. in Steger [u.a.] 1974 erläutert ist $^{6}{ }^{6}$ werden Textsorten als abhängige Variablen der jeweiligen Kommunikationssituation betrachtet. Dabei ist jedes Textexemplar die Realisierung einer Textsorte, jede aktuelle Situation die Realisierung eines Situationstyps (vgl. auch Adamzik 1994). Vor der Textsortenklassifkation steht also eine Situationsklassifikation. Ohne die Problematik der Definition des Situationsbegriffs hier erörtern zu wollen - dazu sei auf Diewald (1991:272-292) verwiesen, wo eine etwas andere Analyse der Situation vorgelegt wird -, ist festzuhalten, daß der Situationsbegriff für eine Gesamtklassifikation auf diejenigen Merkmale eingeschränkt werden muß 3 , die ich die kommunikativen Grundbedingungen nenne. Dies sind keine inhaltlichen Bestimmungen, ${ }^{7}$ sondern rein formale Merkmale der Kommunikationssituation, die a priori bei jeder sprachlichen Kommunikation gegeben sind. ${ }^{8}$ Sie werden aus der Beschaffenheit der prototypischen Kommunikationssituation abgeleitet.

Diese unmarkierte, phylo- und ontogenetisch primäre Grundsituation ${ }^{9}$ jeder Sprachverwendung sei mit Lyons (1983:249) die "kanonische Äußerungssituation" genannt. Sie ist die typische Face-to-face-Situation, in der zwei Partner einen Dialog mit freiem Sprecherwechsel führen. Aus dieser Bestimmung der kanonischen Äußerungssituation lassen sich zwei klassifikationsrelevante Faktoren isolieren, nämlich das dialogische Prinzip und der Partnerkontakt.

Auch Steger 1983; für eine ausführliche Diskussion mit entsprechenden Literaturhinweisen s. Diewald (1991:265ff.).

Anders Harnisch (1990:2103), deren inhaltlich-sozial bestimmter Situationsbegriff u. a. auch Partnerbeziehungen wie Fachmann-Laie einschließt.

Die kommunikativen Grundbedingungen beziehen sich nur auf sprachrelevante Faktoren. Die Bedingungen, die zum Glücken jeglicher Art von Kommunikation vorauszusetzen sind, haben keine differenzierende Kraft. So gehen z. B. Faktoren wie die Existenz von (mindestens) zwei potentiellen Partnern, ihre Bereitschaft zu kommunizieren, ein gemeinsamer Code usw., nicht in die situative Merkmalsmatrix ein. Diese könnte man als Vorbedingungen der sprachlichen Kommunikation bezeichnen. 
Das dialogische Prinzip meint - wie Hartung 1987 sehr überzeugend darlegt ${ }^{10}$ die Tatsache, daß sprachliche Kommunikation grundsätzlich partnerbezogen ist, da sie immer aus dem Gegenüber zweier Perspektiven und deren wechselseitiger Ausrichtung aufeinander besteht. Diese beiden Perspektiven werden von den Kommunikationspartnern eingenommen.

Das dialogische Prinzip zeigt zwei Ausdrucksformen, zwei "Kommunikationsrichtungen" (Brinker 1985:126): Ist es in unmarkierter Form realisiert, liegt ein Dialog vor, seiner markierten Form dagegen entspricht der Monolog. ${ }^{11}$ Das für die Abgrenzung von Dialog und Monolog entscheidende Merkmal ist der Rollenwechsel, also der Austausch der Sprecher- und Hörerrolle bzw. der Produzenten- und Rezipientenrolle. Wie Hartung (1987:100) zurecht betont, ist der Rollenwechsel "weder zufällig noch teleologisch" motiviert. Vielmehr ist er direkter Ausdruck der Art der Realisierung des dialogischen Prinzips. Gleichzeitig ist die Gestaltung des Rollenwechsels ein konstitutives Strukturierungsmittel des Textes. Das dialogische Prinzip ist somit ein situativer Faktor, der über die Realisierung des Wechselmechanismus zu eindeutigen Reflexen im jeweiligen Text führt und daher ein optimales Kriterium für die Textsortenklassifikation darstellt. Es wird hier als das hierarchiehöchste Kriterium zur Einteilung von Texten angesehen (so auch Steger 1983:52 und Weigand 1986:118).

Der Dialog weist realen Wechsel der Sprecher- und Hörerrolle auf. Die Wichtigkeit dieses Merkmals, die schon in Grimms Bezeichnung des Dialogs als "wechselrede der beiden ersten personen" zum Ausdruck kommt (1866:239), wird in allen Definitionen des Dialogs hervorgehoben (vgl. z. B. Henne/Rehbock 1982:14, Mackeldey 1987:38, 42). Nicht so häufig wird jedoch bemerkt, daß dieser Rollentausch zwischen Sprecher und Hörer die konkrete Identifizierbarkeit der beiden Partner füreinander erfordert. ${ }^{12}$ Nur dann ist der den Dialog konstituierende Rollenwechsel ohne Einschränkung vollziehbar. Konkrete Identifizierbarkeit bedeutet die Möglichkeit, ein wechselseitiges Ich

10 Hier wäre eine Vielzahl weiterer Autoren zu nennen. Schon Humboldt (1827/1988:137f.) erwähnt das dialogische Prinzip als Grundlage jeder Sprachverwendung. Tschauder (1986:111) spricht von "Pan-Dialogizität" und auch Hagège 1987 läßt keinen Zweifel an der fundierenden Qualität dieser Eigenschaft; schon der Titel seines Buches "Der dialogische Mensch" bringt seine Grundthese zum Ausdruck, daß "der Merisch [...] von Natur aus dialogisch" ist (244, ähnlich 297). Ähnlich Weigand 1989; ihrer Beschreibung dieses Prinzips als "inhaltliches" und der dazugehörigen sprechakttheoretischen Interpretation (1989:41) kann ich jedoch nicht zustimmen. Das dialogische Prinzip liegt vor jeder inhaltlichen und intentionalen Bestimmung; es ist ausschließlich in der Existenz und Aufeinanderbezogenheit zweier Kommunikationspartner begründet. Aus psychologischer Sicht hält Wygotski (1934/1971:337) fest, daß "die dialogische Sprache tatsächlich die ursprüngliche Sprachform ist." Auch die philosophisch ausgerichtete Untersuchung von Kim 1992 hebt das dialogische Prinzip, das dort unter den "komplementären Charakter" der Kommunikation subsumiert wird, als grụndlegend hervor.

11 Ähnlich Hartung (1987:98), Hagège (1987:259) und Weigand (1989:40), die "zwischen einem formalen, situativen und einem inhaltlichen Begriff des Dialogischen" differenziert.

Weigand 1986 weist darauf hin. 
und $\mathrm{Du}$ zu konstruieren. Wenn dies nicht oder nur eingeschränkt möglich ist, dann ist der Rollenwechsel erschwert und der Dialog nicht mehr in seiner unmarkierten Form realisierbar. Die kanonische Äußerungssituation ist nun als diejenige Situation definiert, die den Dialog in unmarkierter Form, d. h. als gleichberechtigte mündliche Kommunikation zwischen zwei Partnern mit freiem Tausch der Sprecher- und Hörerrolle, ermöglicht. Sie ist also die unmarkierte Kommunikationssituation, der die unmarkierte Form der Sprachverwendung entspricht.

Der Partnerkontakt als der zweite textsortenrelevante Faktor der kommunikativen Grundbedingungen kann in mehrere Aspekte untergliedert werden. Er betrifft die Zahl der Beteiligten, ihre soziale Beziehung und ihre räumliche und zeitliche Beziehung. Das Gemeinsame dieser Aspekte des Partnerkontakts ist, daß sie Einfluß auf die Konkretheit der Kommunikationspartner füreinander - und daher auf den Wechselmechanismus - haben. Wenn z. B. die Zahl der Beteiligten größer als zwei ist und somit eine markierte Situation vorliegt, verkompliziert sich der Wechselmechanismus. Auch der soziale Faktor des Bekanntheitsgrades der Partner beeinflußt die Marktiertheit der Situation und damit die Komplexität des Wechselmechanismus.

Es ist durchaus denkbar, daß noch weitere Aspekte des Partnerkontakts berïcksichtigt werden müßten, daß also an dieser Stelle die ansonsten eher minimalistische situative Merkmalsmatrix erweiterungsfähig ist. Die folgenden Ausführungen werden sich jedoch auf die raum-zeitliche Ausprägung des Partnerkontakts konzentrieren, da sie die deutlichsten Textreflexe erzeugt.

Bezogen auf die raum-zeitliche Einordnung realisiert sich der Partnerkontakt entweder als direktes Gegenüber (gleichbedeutend mit "face-to-face"), d. h. als Ortsund Zeitgleichheit der Partner, oder als Situation, in der Ortsgleichheit und/oder Zeitgleichheit nicht gegeben sind. Dazu ist anzumerken, daß selbst bei diesem scheinbar objektiv definierten Faktor der Äußerungssituation die Situationsinterpretation durch die Teilnehmer eine große Rolle spielt (dazu auch Harnisch 1988:46). Innerhalb gewisser Grenzen steht es im Ermessen der Kommunikationspartner, zu beurteilen, ob sie sich am gleichen Ort und zur gleichen Zeit befinden oder nicht.

Die raum-zeitlichen Gegebenheiten beeinflussen den Wechselmechanismus und damit die Textgestalt, insofern, als bei einer Abweichung von ihrer unmarkierten Realisierung der Wechselmechanismus verkompliziert wird. Allerdings muß beachtet werden, daß die Ausprägung der Kommunikationsrichtung als Dialog oder Monolog von den raum-zeitlichen Gegebenheiten zwar in ihrer Gestalt beeinflußt, aber nicht grundsätzlich determiniert wird. Die Konkretheit der Partner füreinander, die ja entscheidend für die Art der Realisierung des dialogischen Prinzips ist, setzt sich aus mehr als nur raum-zeitlichen Bedingungen zusammen.

Direkten Einfluß auf die Textgestalt hat die Raum-Zeit-Komponente des Partnerkontakts jedoch im Hinblick auf das Medium. Unter Medium verstehe ich die Realisierung eines Textes in akustisch-auditiver also gesprochener bzw. mündlicher oder in 
optisch-visueller also geschriebener bzw. schriftlicher Form. Die mediale Ausprägung eines Textes ist also ein Textmerkmal, das von der Ausprägung eines situativen Merkmals, nämlich des raum-zeitlichen Partnerkontakts, determiniert wird.

Die Orts- und Zeitgleichheit der Partner führt im Normalfall zur Realisierung des Textes als mündlich. Daß Situationen, die markierte Werte für den raum-zeitlichen Partnerkontakt aufweisen, also Ort- und Zeitungleichheit der Partner, überhaupt als Äußerungssituationen gelten können, ist technischen Hilfsmitteln zu verdanken, die die Kluft zur unmarkierten Situation überbrücken. Kim (1992:14) spricht von diesen Mitteln als "Fortsetzungen und erweiterte Formen des zwischenmenschlichen 'Gesprächs'". Sie erfordern zusätzlichen Aufwand vom Individuum und der gesamten Gesellschaft und erzeugen markierte Äußerungssituationen und entsprechend markierte Grundtextsorten. Bei Ort- und Zeitungleichheit erfolgt die Realisierung des Textes im Normalfall als schriftlich. Man kann die Schrift, d. h. die Vermittlung über den optischvisuellen Kanal, geradezu als das Standardmittel zur kommunikativen Bewältigung dieser markierten Äußerungssituation betrachten.

Die Entwicklung neuer Kanäle, d. h. neuer technischer Träger, z. B. die Bandaufnahme, die verschickt und an einem anderen Ort zu anderer Zeit abgehört werden kann (der Tonbandbrief), führt dazu, daß nun auch der akustisch-auditive Kanal bei Ort- und Zeitungleichheit offen ist und der Text daher mündlich realisiert werden kann. Allerdings ist zu vermuten, daß gesprochene Sprache, die von vorne herein zum Zweck der konservierenden Aufzeichnung geäußert wird - Dimter (1981:43f.) spricht von Texten, die schon in ihrer "Originalsituation" als "Konserven" geplant sind -, durch die Aufhebung ihrer Flüchtigkeit letztlich dem schriftlichen Medium näher ist als der gesprochenen Sprache, die nur zum einmaligen Hören geäußert wird. Eventuell müßte man also beim Medium statt zwischen mündlich und schriftlich, zwischen flüchtig und konserviert unterscheiden. Obwohl dies auf lange Sicht vielleicht die bessere Lösung sein wird, bleibe ich bei den eingeführten Termini mündlich versus schriftlich bzw. gesprochen versus geschrieben.

Bis zur Erfindung des Telefons kovariierten Ortsgleichheit und Zeitgleichheit miteinander. Eine Situation war entweder face-to-face oder sie war es nicht. Beim Telefon, das die Zeitgleichheit trotz Ortsungleichheit erlaubt, entsteht ein im echten Sinn gesprochener Text, $d . h$. die Sprache ist nicht konserviert, sondern wird direkt im akustisch-auditiven Kanal übermittelt und als gesprochene Sprache realisiert.

Als Zusammenfassung dieses Abschnitts sei festgehalten, daß das dialogische Prinzip und der Partnerkontakt situative Faktoren sind, die in jeder Situation, in der sprachliche Kommunikation stattfindet, in der einen oder anderen Ausprägung realisiert sind. Sie werden hier als die kommunikativen Grundbedingungen bezeichnet und liegen, als objektive Gegebenheiten der Situation, vor den bewußten oder unbewußten Absichten der Sprecher. Sie sind insofern unhintergehbar und für den Beobachter leichter zugänglich als z. B. intentionale Faktoren. Für beide situativen Faktoren gilt ferner, daß ihre verschiedenen Ausprägungen sich auf die Gestaltungsmöglichkeit jedes 
Textes auswirken. Die Ausprägung des dialogischen Prinzips führt zur Realisierung des Textes als Dialog oder Monolog, d. h. zur Durchführung oder Unterlassung des Rollenwechsels. Die verschiedenen Ausprägungen des Partnerkontakts haben Einflu $\beta$ auf die Art der Durchführung des Rollenwechsels. Außerdem - und das ist entscheidender beeinflussen sie die Wahl des Mediums, also die Realisierung des Textes als mündlich oder schriftlich. Im folgenden wird gezeigt, wie verschiedene Kombinationen der Ausprägungen der situativen Grundbedingungen zu verschiedenen Kombinationen von Textmerkmalen, also zu verschiedenen Grundtextsorten, führen.

\section{Grundtextsorten}

Der Situationstyp der kanonischen Äußerungssituation wurde als die für sprachliche Kommunikation unmarkierte, natürliche Situation bezeichnet. Das dialogische Prinzip und der raum-zeitliche Partnerkontakt sind jeweils in ihrer unmarkierten Form, d. h. als Dialog und als Ort- und Zeitgleichheit realisiert. Die dieser Situation entsprechende Grundtextsorte, ich nenne sie auch Grundtextsorte I, ist der direkte Dialog, dessen sprachliche Strukturierung vom real vollzogenen Sprecherwechse ${ }^{13}$ und vom mündlichen Medium geprägt ist. Man kann sagen, der Dialog ist die unmarkierte Grundtextsorte der unmarkierten Äußerungssituation.

Texte mit markierter Ausprägung der Dialogizität sind auf eine markierte Sprechsituation zurückzuführen, also eine Sprechsituation, die von der kanonischen Äußerungssituation abweicht. Die markierteste Äußerungssituation ist diejenige, in der alle Merkmale abweichend von ihrer Ausprägung in der kanonischen Äußerungssituation besetzt sind. Das dialogische Prinzip ist als Monolog ausgeprägt, der Partnerkontakt ist nur mehr minimal konkret, was für seine raum-zeitliche Komponente Orts- und Zeitungleichheit bedeutet. Die diesem markierten Situationstyp entspringenden Texte werden zur Grundtextsorte V, zum schriftlichen Monolog, zusammengefaßt. Der schriftliche Monolog ist die am stärksten markierte Grundtextsorte. Seine Textstruktur ist vom Fehlen des Rollenwechsels und vom schriftlichen Medium geprägt. Dieser Grundtextsorte gehören die meisten Texte an, die sich in Büchern, Zeitschriften, Zeitungen usw. finden. Schriftliche Monologe tendieren dazu, jeglichen Hinweis auf den außersprachlichen Kontext aus dem Text zu tilgen und in den Textraum zu verlagern. Unter Textraum verstehe ich den aus sprachlichen und nichtsprachlichen Elementen bestehenden Rahmen (z. B. Überschriften, Bilder usw.) schriftlicher Monologe, der die Rekonstruktion der Situation durch den Rezipienten ermöglicht. Mit ähnlicher Zielrichtung spricht Lerchner (1990:324) hier von der "Selbstkontextualisierung" der Texte, die ein Ausdruck von zunehmender Markiertheit und abnehmender Situationsanbin- 
dung ist. Der Grundtextsorte V gehört eine Vielzahl von Textsorten an, die sich z. T. stark darin unterscheiden, wie vollständig die Kommunikationssituation aus dem Text verdrängt wurde.

Zwischen den beiden Endpunkten der Skala, dem direkten Dialog und dem schriftlichen Monolog, unterscheide ich drei weitere Grundtextsorten, die durch verschiedene Kombinationen der Realisierung des dialogischen Prinzips und des Partnerkontakts entstehen. Als deren prototypische Vertreter können das Tefelongespräch, der Brief und der mündliche Monolog gelten.

Das Telefongespräch vertritt die Grundtextsorte II, die dem direkten Dialog am nächsten steht. Die ihm zugrundeliegenden situativen Faktoren sind die unmarkierte Ausprägung des dialogischen Prinzips und die teilweise markierte Ausprägung des Partnerkontakts, d. h. Zeitgleichheit bei Ortsungleichheit der Partner. Das Telefongespräch kann einerseits als ein der visuellen Komponente beraubter Dialog, andererseits als ein durch technische Mittel in Richtung Dialog optimierter Brief bezeichnet werden. Die damit korrelierenden Texteigenschaften sind die mündliche Realisierung und der reale Rollenwechsel, dessen spezifische Komplikationen direkt auf das Fehlen des visuellen Kontakts, also auf die Ortsungleichheit, zurückführbar sind (Diewald 1987).

Der Brief als Prototyp der Grundtextsorte III nimmt die mittlere Position auf der Skala der fünf Grundtextsorten ein. Seine situative Merkmalsmatrix zeigt die unmarkierte Ausprägung des dialogischen Prinzips und markierten Partnerkontakt, nämlich Orts- und Zeitungleichheit. Aus der Ausprägung des Partnerkontakts ergibt sich die mediale Realisierung des Textes als schriftlich. Kontrovers wird jedoch die Frage beantwortet, ob der Brief bzw. der Briefwechsel im eigentlichen Sinn dialogisch ist oder nicht.

Ermerts Ausweg (1979:62), den Brief bezüglich der Kommunikationsrichtung als "neutral" zu bezeichnen, ist keine Lösung, da die Definition des dialogischen Prinzips es verlangt, festzulegen, ob ein Wechsel und damit ein Dialog stattfindet oder nicht. Brinker 1985 bezeichnet den Brief als monologisch und hebt die Abgeschlossenheit und Selbständigkeit eines Einzelbriefes hervor (127, Anm. 25). ${ }^{14}$ Hier dagegen wird der Brief mit Weigand ${ }^{15}$ als dialogisch betrachtet, und zwar aus folgenden Gründen: Beide Partner haben die Absicht, als füreinander. (wie gut auch immer) bekannte und identifizierte Individuen zu kommunizieren und erwarten einen mindestens einmaligen Rollenwechsel. Dieser ist jedoch durch raum-zeitliche Faktoren behindert, d. h. nicht als freie Wechselrede möglich. Der Brief ist damit ein versuchter, ein rudimentärer Dialog.

14 Ähnlich Tschauder (1986:110), der den Einzelbrief als monologisch, den Brief und die Antwort gemeinsam als dialogisch betrachtet.

15 Weigand (1989:42, Anm. 42) und (1986:119): "Ein Einzelbrief darf daher nicht isoliert werden, ist nicht als monologisch zu bezeichnen." 
Die Grundtextsorte IV, die ich auch als mündlichen Monolog bezeichne, umfaßt Vorträge, Reden und dergleichen. Thre situativen Merkmale sind monologisch und ortund zeitgleich. Wenn der Brief als ein den raum-zeitlichen Umständen trotzender Dialogversuch beschrieben wird, so ist der mündliche Monolog sein Gegenstück, nämlich die Einschleusung eines (schriftlich vorbereiteten) Monologs in eine Face-to-face-Situation. Auch hier gibt es viele Zwischenstufen und Variationen, doch der prototypische mündliche Monolog ist vorher entworfen, meist sogar schriftlich fixiert und schließt eine Wechselrede aus.

Dennoch ist der mündliche Monolog eine deutlich ausgeprägte und vom schriftlichen Monolog abgesetzte Grundtextsorte. Die unmarkierte Ausprägung des Partnerkontakts als zeit- und ortsgleich ist von so großem Gewicht, daß in jedem mündlichen Monolog, wenn auch nur an dafür vorgesehenen, ritualisierten Stellen, auf die Situation Bezug genommen werden muß. Diese Grundtextsorte hält als Monolog dem Dialogdruck der Face-to-face-Situation stand, was dadurch begünstigt wird, daß die Vielzahl der Zuhörer (d. h. potentieller Dialogpartner) die markierte Ausprägung einer Komponente des Partnerkontakts darstellt.

Theoretisch sind weitere Grundtextsorten denkbar, vor allem aufgrund der Tatsache, daß durch die Entwicklung neuer technischer Kanäle neue Möglichkeiten der Kombination der Merkmale des Partnerkontakts gegeben sind. Dies bedeutet, daß sich das Inventar der Grundtextsorten verschieben bzw. erweitern kann. Hier ist jedoch zum einen die Einschränkung zu machen, daß die oben erwähnten Beispiele nur die prototypischen Vertreter einer Grundtextsorte darstellen und daß neue Kombinationen der situativen Faktoren womöglich nur zu Textsorten führen, die als periphere Mitglieder bereits bestehender Grundtextsorten eingeordnet werden können (z. B. Tonbandbrief, Bildtelefon). Zum anderen sollte man die bloße Möglichkeit der Merkmalskombinatorik nicht allein als konstitutiv für eine Grundtextsorte betrachten, sondern auch die Forderung nach allgemeiner Gebräuchlichkeit und Zugänglichkeit erheben. Ein letztes Argument für die Auffassung, daß die dargestellte Skala der fünf Grundtextsorten eine im wesentlichen stabile Gliederung ist, ergibt sich aus der Tatsache, daß die Grundtextsorten aus den Realisierungen der kommunikativen Grundbedingungen abgeleitet sind und daß diese - wenn sprachliche Kommunikation funktionieren soll nicht beliebig veränderbar sind. Mit der vorgestellten Skala dürften also die wesentlichen Prototypen erfaßt sein, so daß fundamentale Änderungen des Bestandes nicht zu erwarten sind. 16 den mündlichen Monolog reduzieren, es würden jedoch auch hier keine völlig anderen Grundtextsorten auftreten. 


\section{Textsortenklassifikation und Textmerkmale}

In den letzten beiden Abschnitten wurde gezeigt, daß die kommunikativen Grundbedingungen, also das dialogische Prinzip und der Partnerkontakt, eindeutige Textreflexe verursachen. Die Ausprägung des dialogischen Prinzips als Dialog oder Monolog wirkt massiv auf die Strukturierung des Textes, die Art des raum-zeitlichen Partnerkontakts wirkt sich vor allem auf seine mediale Gestaltung aus. Diese Unterschiede wurden zur Konstituierung der Grundtextsorten herangezogen.

Der Einfluß der kommunikativen Grundbedingungen beschränkt sich jedoch nicht auf diese globalen Textmerkmale, sondern er läßt sich auch in der Mikrostruktur des Textes an bestimmten sprachlichen Elementen nachweisen. Dies sind die deiktischen Markierungen, ${ }^{17}$ deren Bezug zu den situativen Faktoren man sich folgendermaßen verdeutlichen kann: Der Prozeß der Deixis ist umschreibbar als im Sprecher verankertes "sprachliches Zeigen" auf außersprachliche Kontextelemente. Der Sprecher bezieht sich von seinem Standpunkt, von - mit Bühler (1934/1982:102f.) gesprochen - seiner Origo aus, auf bestimmte Elemente des Äußerungskontexts, indem er sie relativ zu seinem Standpunkt lokalisiert. Das Gelingen dieser Lokalisierung ist direkt abhängig von den Gegebenheiten der aktuellen kommunikativen Situation. Ein deiktischer Ausdruck wie hier kann vom Rezipienten nur richtig interpretiert werden, wenn er die situativen Gegebenheiten kennt. Die Verwendung von hier ist also nur dann sinnvoll, wenn diese Kenntnis vorausgesetzt werden kann. Allgemeiner formuliert: Da Deiktika auf Kontextelemente verweisen, ist ihr Gebrauch nur in denjenigen kommunikativen Situationen angemessen, die den Zugang zu diesen Kontextelementen erlauben. Das Auftreten oder Fehlen von Deiktika in einem Text reflektiert daher die Kommunikationssituation, in der er produziert wurde, d. h. Deiktika sind direkte Indikatoren bestimmter situativer Merkmale und damit der von diesen Merkmalen gesteuerten Textsorte.

Ein Teil der deiktischen Markierungen reagiert auf die Art der Ausprägung des Partnerkontakts, und hier wiederum besonders auf seine raum-zeitliche Komponente. Dies sind bestimmte lokale und temporale Deiktika sowie Deiktika, die die Referenz auf Gegenstände und sogenannte "dritte Personen" herstellen. Die Ausprägung des dialogischen Prinzips dagegen wird nur von einigen wenigen Deiktika reflektiert, die aber von großer Bedeutung für die Textsortendifferenzierung sind. Es handelt sich um die Personalformen der ersten und zweiten Person, die ja keine Pronomina, sondern - mit Braunmüller (1977:23) gesprochen - "Rollenvariablen" sind. Sie verweisen nicht auf eine Nominalphrase, sondern sie referieren auf den Sprecher bzw. den Hörer und wechseln ihre Referenz im Gleichklang mit dem Rollenwechsel. Ihre Verwendung und Verteilung ist nicht von der räumlichen und zeitlichen Ausprägung des Partnerkontakts

17 Die Textsortensensitivität von Deiktika wurde schon mehrfach erkannt und auch zur Textsortenbestimmung herangezogen. Dies geschah allerdings nur in Bezug auf einzelne Textsorten und nicht systematisch; hierzu und zu den entsprechenden Literaturhinweisen s. Diewald (1991:264). 
abhängig, sondern allein von der Ausprägung des dialogischen Prinzips (s. dazu Diewald 1991:202-226).

Ohne hier auf Details eingehen zu können, möchte ich kurz auf eine exemplarische Analyse in Diewald 1991 hinweisen, die die textsortendifferenzierende Potenz der Rollenvariablen untersucht. Sie beansprucht keine empirische Relevanz, ihre Ergebnisse zeigen aber doch, daß eine weitere Suche in dieser Richtung lohnend sein könnte. Pro Grundtextsorte wurde ein Beispieltext im Hinblick auf die Frequenz und Distribution verschiedener deiktischer Mittel analysiert. Die unterschiedlichen Werte wurden als textuelle Reflexe der die Grundtextsorten konstituierenden situativen Merkmale interpretiert.

Insgesamt ergab sich, daß die Grundtextsorten bezüglich der Gesamtzahl der Deiktika zwei Gruppen bilden: Dialogische Grundtextsorten zeigen einen deutlich höheren Anteil von Deiktika an der Gesamtwortzahl (über $8 \%$ ) als monologische Grundtextsorten (unter 5\%). Diese hohe Zahl bei den dialogischen Grundtextsorten ist auf den überproportionalen Anteil der Personalformen an der Summe der Deiktika zurückzuführen. Monologische Grundtextsorten weisen dagegen wenige oder gar keine personalen Deiktika auf. Dieser Befund bekräftigt die Relevanz des dialogischen Prinzips und rechtfertigt seine Einschätzung als dominanter Faktor der kommunikativen Grundbedingungen. Indirekt bestätigt wird er auch durch Gläser 1990. Sie untersucht nur monologische Fachtextsorten, stellt keinerlei Verbindung zwischen den Rollenvariablen und der Kommunikationsrichtung her und hat auch nicht das Ziel, die kommunikativen Grundbedingungen als relevante Klassifikationsbasis zu erweisen. Obwohl also ihr Erkenntnisinteresse in eine andere Richtung zielt, kommt sie zu dem Ergebnis, daß im Gegensatz zu allen anderen von ihr untersuchten sprachlichen Merkmalen (Passiv, rhetorische Figuren), gerade die "Verwendung der Pronomen der 1. Person Singular und Plural sowie der 2. Person" eine textsortenrelevante Distribution aufweist (Gläser 1990:301 und passim).

Auch die zweite Merkmalsachse, der Partnerkontakt, zeigt direkte Reflexe in der Frequenz und Distribution der Rollenvariablen, allerdings in zunächst unerwarteter Weise. Auf die Gesamtwortzahl bezogen weist das Telefongespräch mit rund 13\% den höchsten Anteil an personaler Deixis auf. Thm folgen in deutlichem Abstand der Brief mit rund 7\% und der direkte Dialog mit 5\%. Die Erklärung für die zunächst erstaunliche Tatsache, daß Telefongespräch und Brief höhere:Werte an Personaldeiktika aufweisen als der Dialog der kanonischen Äußerungssituation, liegt in der Beschaffenheit des raum-zeitlichen Partnerkontakts. Da beim Dialog direkter Kontakt zwischen den Partnern besteht, kann vieles implizit bleiben, was beim Telefondialog und beim Brief expliziert, d. h. versprachlicht, werden muß. So sind z. B. sowohl für das Telefongespräch als auch für den Brief stark normierte Rituale der Kontaktsteuerung - wie Anfangs-, Gruß- und Beendigungsrituale - obligatorisch, die einen hohen Anteil personaler Deixis enthalten. Beim Telefongespräch muß außerdem der Dialogrollenwechsel unter der markierten Bedingung der Ortsungleichheit der Partner stattfinden, was die Frequenz der Personaldeiktika ebenfalls erhöht. D. h. markiertere Formen des Partner- 
kontakts bei gleichzeitiger Realisierung des Dialogs führen zu einer im Vergleich zum unmarkierten Dialog starken Zunahme an personaler Deixis.

Insgesamt unterscheiden sich die untersuchten Texte als Repräsentanten der fünf Grundtextsorten deutlich in der Frequenz und Distribution der auftretenden Deiktika. Die hier behandelten Unterschiede lassen sich direkt auf die Ausprägungen der Merkmale der kommunikativen Grundbedingungen zurückführen.

\section{Schlußbemerkung}

Die obigen Ausführungen haben gezeigt, daß die kommunikativen Grundbedingungen relevante Klassifikationsmerkmale darstellen, die eine nichtwillkürliche Textsortenklassifikation erlauben. Zum einen sind sie universell auf jedes Textvorkommen applizierbar, zum andern verursachen ihre Varianten direkte Reflexe im Text, und zwar sowohl in seiner globalen Struktur und medialen Gestaltung, als auch bei den deiktischen Markierungen, vor allem bei den Rollenvariablen. Die diese Affinitäten widerspiegelnde Klassifikation der Grundtextsorten ist $\mathrm{m}$. E. die Basis, auf der eine weitere Differenzierung der Textsorten erfolgen kann. In Diewald 1991 werden einige weitere Klassifikationsebenen benannt, die gesellschaftliche, funktionale und thematische Faktoren berücksichtigen. Ohne dies weiter auszuführen, möchte ich auf einen wichtigen Unterschied hinweisen, der zwischen diesen Faktoren und den kommunikativen Grundbedingungen besteht. Letztere stellen die nichthintergehbaren Grundfaktoren der Textproduktion dar, während erstere die Motive für die Texterzeugung liefern. Man will über ein Thema kommunizieren, eine Intention verwirklichen, oder man fühlt sich durch den sozialen Kontext zur Textproduktion veranlaßt. Aus der Situation dagegen lassen sich keine Motive zur Textproduktion ableiten. Sie ist vielmehr deren Grundlage und nur innerhalb der von ihr gesteckten Grenzen können die anderen Ebenen realisiert werden. Die Merkmalsausprägung der hierarchieniedrigeren Ebenen wirkt also subklassifizierend auf der Basis der Grundtextsorten. Das hier vorgeschlagene Modell ermöglicht also eine Klassifikation mit variablem Differenzierungsgrad, die bei Bedarf auch historisch-individuelle Textsortennormen erfassen kann und dennoch immer in die Gesamtklassifikation der Grundtextsorten integriert bleibt.

\section{LITERATUR}

Adamzik, Kirsten: Forschungsstrategien im Bereich der Textsortenlinguistik. In: Zeitschrift für Germanistik, Neue Folge 1, 1991, S. 99-109.

Adamzik, Kirsten: Zum Textsortenbegriff am Beispiel von Werbeanzeigen. In: Satz Text - Diskurs. Akten des 27. Linguistischen Kolloquiums, Münster 1992, Bd. 2. Hg. Peter-Paul König und Helmut Wiegers. Tübingen 1994, S. 173-180.

Bahner, Werner, Joachim Schildt und Dieter Viehweger (Hg.): Proceedings of the Fourteenth International Congress of Linguists: Berlin/GDR, August 10 - August 15, 1987, Bd. 3, Berlin 1990. 
Braunmüller, Kurt: Referenz und Pronominalisierung. Zu den Deiktika und Proformen des Deutschen. Stuttgart 1977.

Brinker, Klaus: Linguistische Textanalyse. Eine Einführung in Grundbegriffe und Methoden. Berlin 1985.

Bühler, Karl: Sprachtheorie. Die Darstellungsfunktion der Sprache. Mit einem Geleitwort von Friedrich Kainz. Stuttgart 1982. [Nachdruck von 1934].

Coseriu, Eugenio: Synchronie, Diachronie und Geschichte. Das Problem des Sprachwandels. Übersetzt von Helga Sohre. München 1974. [Span. Original 1958].

Diewald, Gabriele: Telefondialoge. Beschreibungsversuche zwischen Gesprächsanalyse und Textlinguistik. Zulassungsarbeit zur Ersten Staatsprüfung für das Lehramt an Gymnasien. Erlangen 1987. [Typoskript].

Diewald, Gabriele: Deixis und Textsorten im Deutschen. Tübingen 1991.

Dimter, Matthias: Textklassenkonzepte heutiger Alltagssprache. Kommunikationssituation, Textfunktion und Textinhalt als Kategorien alltagssprachlicher Textklassifikation. Tübingen 1981.

Ehlich, Konrad: Die Entwicklung von Kommunikationstypologien und die Formbestimmtheit des sprachlichen Handelns. In: Kallmeyer, Werner (Hg.), 1986, S. 47-72.

Ermert, Karl: Briefsorten. Untersuchungen zur Theorie und Empirie der Textklassifikation. Tübingen 1979.

Franke, Wilhelm: Texttypen - Textsorten - Textexemplare. Ein Ansatz zu ihrer Klassifizierung und Beschreibung. In: Zeitschrift für Germanistische Linguistik, 15, 1987, S. 263-281.

Franke, Wilhelm: Linguistische Texttypologie. In: Aspekte der Textlinguistik. Hg. Klaus Brinker. Germanistische Linguistik, 106-107, Hildesheim [usw.] 1991, S. 157-182.

Fröhlich, Ilse: Kriterien für eine Klassifizierung nichtoffizieller Gespräche. In: Bahner, Werner, Joachim Schildt und Dieter Viehweger (Hg.), 1990, S. 2079-2082.

Gläser, Rosemarie: Fachtextsorten im Englischen. Tübingen 1990.

Grimm, Jakob: Ueber den Personenwechsel in der Rede. In: Abhandlungen zur Literatur und Grammatik. Kleinere Schriften, Bd. 3, Berlin 1866, S. 236-311.

Gülich, Elisabeth: Textsorten in der Kommunikationspraxis. In: Kallmeyer, Werner (Hg.), 1986, S. 15-46.

Hagège, Claude: Der dialogische Mensch. Sprache - Weltbild - Gesellschaft. Aus dem Französischen von Monika Hübner. Reinbek bei Hamburg 1987. [Frz. Original 1985].

Harnisch, Hanna: Situativität und Textsorte. In: Funktionalkommunikative Aspekte des Sprachsystems und des Textes. Hg. Hanna Harnisch und Georg Michel. Potsdamer Forschungen der Pädagogischen Hochschule "Karl Liebknecht", Gesellschaftswissenschaftliche Reihe, Heft 89, Potsdam 1988, S. 44-54.

Harnisch, Hanna: Kommunikationssituation und textsortenspezifische Sprachverwendung. In: Bahner, Werner, Joachim Schildt und Dieter Viehweger (Hg.), 1990, S. 2101-2103. 
Hartung, Wolfdietrich: Das Dialogische als Prinzip des Sprachlichen. Positionen zwischen Kontinuität und Diskontinuität. In: Bedeutungen und Ideen in Sprachen und Texten. Werner Bahner gewidmet. Hg. Werner Neumann und Bärbel Techtmeier. Berlin 1987, S. 93-111.

Henne, Helmut und Helmut Rehbock: Einführung in die Gesprächsanalyse. 2. verb. u. erw. Aufl. Berlin, New York 1982.

von Humboldt, Wilhelm: Ueber den Dualis. In: Schriften zur Sprachphilosophie. Werke in fünf Bänden, Bd. 3. Hg. Andreas Flitner und Klaus Giel. 6. unveränderte Aufl. Darmstadt 1988, S. 113-143. [Zuerst 1827].

Hundsnurscher, Franz und Edda Weigand (Hg.): Dialoganalyse. Referate der 1. Arbeitstagung Münster 1986. Tübingen 1986.

Kallmeyer, Werner (Hg.): Kommunikationstypologie. Handlungsmuster, Textsorten, Situationstypen. Jahrbuch 1985 des Instituts für deutsche Sprache. Düsseldorf 1986. Kim, Tack-Whan: Realitätsprinzipien und Kommunikationsmodelle. Eine Studie zur Grundlegung der notwendigen Bedingungen der zwischenmenschlichen Kommunikation und zum Aufbau von Kommunikationsmodellen in der Wirklichkeit. Münster 1992.

Lerchner, Gotthard: Kontextualisierung historischer Texte. Zum Markiertheitsprinzip in einer textsortenbezogenen Sprachhistoriographie. In: Zeitschrift für Phonetik, Sprachwissenschaft und Kommunikationsforschung, 43, 1990, S. 315-326.

Lux, Friedemann: Text, Situation, Textsorte. Probleme der Textsortenanalyse, dargestellt am Beispiel der britischen Registerlinguistik. Mit einem Ausblick auf eine adäquate Textsortentheorie. Tübingen 1981.

Lyons, John: Semantik, Bd 2. Aus dem Englischen übertragen und für den deutschen Leser eingerichtet von Jutta Schust. München 1983. [Engl. Original 1977].

Mackeldey, Roger: Alltagssprachliche Dialoge. Kommunikative Funktionen und syntaktische Strukturen. Leipzig 1987.

Rolf, Eckard: Die Funktionen der Gebrauchstextsorten. Berlin, New York 1993.

Steger, Hugo [u. a.]: Redekonstellation, Redekonstellationstyp, Textexemplar, Textsorte im Rahmen eines Sprachverhaltensmodells. In: Gesprochene Sprache. Jahrbuch 1972. Sprache der Gegenwart, 26, Düsseldorf 1974, S. 39-97.

Steger, Hugo: Über Textsorten und andere Textklassen. In: Textsorten und literarische Gattungen. Dokumentation des Germanistentages in Hamburg vom 1. bis 4. April 1979. Hg. Vereinigung der deutschen Hochschulgermanisten. Berlin 1983, S. $25-$ 67.

Tschauder, Gerhard: Dialogende und Textende. In: Hundsnurscher, Franz und Edda Weigand (Hg.), 1986, S. 103-113.

Weigand, Edda: Dialogisches Grundprinzip und Textklassifikation. In: Hundsnurscher, Franz und Edda Weigand (Hg.), 1986, S. 115-125.

Weigand, Edda: Sprache als Dialog. Sprechakttaxonomie und kommunikative Grammatik. Tübingen 1989. 
Weigand, Edda: Mündlich und schriftlich - ein Verwirrspiel. In: Dialoganalyse IV. Referate der 4. Arbeitstagung Basel 1992, Teil 1. Hg. Heinrich Löffler unter Mitarbeit von Christoph Grolimund und Mathilde Gyger. Tübingen 1993, S. 137-150. Wierzbicka, Anna: Cross-Cultural Pragmatics. The Semantics of Human Interaction. Berlin, New York 1991.

Wygotski, Lew Semjonowitsch: Denken und Sprechen. Mit einer Einleitung von Thomas Luckmann. Hg. Johannes Helm. Übers. von Gerhard Sewekow. Frankfurt am Main 1971. [Russ. Original 1934].

\section{Zusammenfassung}

Es wird eine integrative Basisklassifikation vorgeschlagen, die es ermöglicht, alle Textvorkommen einer von fünf Grundtextsorten zuzuordnen und die zudem auf nichtwillkürlichen Klassifikationsmerkmalen beruht. Die Grundtextsorten sind ausschließlich durch situative Merkmale bestimmt und bilden die Basis für eine feinere Differenzierung nach funktionalen, inhaltlichen und soziohistorischen Kritierien. Die klassifikationsrelevanten situativen Merkmale werden aus den kommunikativen Grundbedingungen abgeleitet, $d$. h. aus Bedingungen, die die Grundlage und Voraussetzung für jede Textproduktion bilden und daher als universell betrachtet werden können. Aus diesen Grundbedingungen werden zwei Klassifikationsparameter isoliert, nämlich das dialogische Prinzip und der Partnerkontakt. Mit ersterem ist die grundsätzliche Partnerbezogenheit der sprachlichen Kommunikation, d. h. die Gegenüberstellung zweier Perspektiven, erfaßt; letzterer bezieht sich vor allem auf die raum-zeitliche Beschaffenheit der kommunikativen Situation. Die verschiedenen Kombinationen dieser situativen Faktoren bilden die Situationstypen für die entsprechenden Grundtextsorten. Letztere sind in einer Skala zunehmender Markiertheit angeordnet, die mit der zunehmenden Markiertheit des jeweiligen Situationstyps korreliert. Der unmarkierten Kommunikationssituation - das ist die dialogische Face-to-face-Situation zweier Partner - entspricht die unmarkierte Grundtextsorte, der direkte Dialog; am markierten Ende der Skala steht der schriftliche Monolog, dem der maximal markierte Situationstyp entspricht, der in allen Merkmalen vom unmarkierten Situationstyp abweicht. Die Grundtextsorten weisen distinktive sprachliche Merkmale auf, die direkt auf die jeweilige Ausprägung der situativen Gegebenheiten zurückführbar sind.

\section{Povzetek}

\section{KLASIFIKACIJA BESEDILNIH VRST NA OSNOVI TEMELJNIH SPOROČANJSKIH POGOJEV}

Avtorica predlaga osnovno integrativno klasifikacijo, s katero je mogoče vse pojavne oblike besedil uvrstiti $v$ eno od petih temeljnih besedilnih vrst in ki temelji na danih klasifikacijskih značilnostih. Temeljne besedilne vrste so določene izključno s situacijskimi značilnostmi, ki oblikujejo osnovo za natančnejšo diferenciacijo glede na fukcionalne, vsebinske in sociozgodovinske kriterije. Situacijske značilnosti, relevantne za oblikovanje klasifikacije, so izpeljane iz osnovnih sporočanjskih pogojev, t.j. iz pogojev, ki so osnova in pogoj za vsako tvorjenje besedila in ki jih zato pojmujemo kot univerzalne. Iz teh osnovnih pogojev sta izvzeta dva klasifikacijska parametra, in sicer dialoški princip in stik s partnerjem. Prvi zajema načelno naravnanost jezikovnega sporočanja na partnerja, t.j. soočenje dveh perspektiv; drugi kriterij se nanaša predvsem na prostorsko-časovno strukturo sporočanjske situacije. Različne kombinacije ustrezne besedilne vrste. Slednje so razvrščene v lestvici rastoče označenosti, ki je povezana $z$ rastočo označenostjo vsakokratnega situacijskega tipa. Neoznačeni sporočanjski situaciji - to je dialoška situacija "face-to-face" dveh partnerjev - ustreza neoznačena temeljna besedilna vrste, t.j. neposredni dialog; na označenem koncu lestvice je pisni monolog, ki mu ustreza najbolj označen situacijski tip, ki $v$ vseh značilnostih odstopa od neoznačenega situacijskega tipa. Temeljne besedilne vrste vsebujejo razločevalne jezikovne značilnosti, s katerimi je mogoče neposredno razložiti vsakokratno izraznost situacijske danosti. 